\title{
Pair correlation of the zeros of the Riemann zeta function in longer ranges
}

by

\author{
Tsz Ho Chan (Cleveland, OH)
}

1. Introduction. We assume the Riemann Hypothesis $(\mathrm{RH})$ for the Riemann zeta function $\zeta(s)$ throughout this paper, thus $\varrho=1 / 2+i \gamma$ denotes a non-trivial zero of the Riemann zeta function.

In the early 1970s, Hugh Montgomery considered the pair correlation function

$$
F(x, T)=\sum_{0<\gamma, \gamma^{\prime} \leq T} x^{i\left(\gamma-\gamma^{\prime}\right)} w\left(\gamma-\gamma^{\prime}\right) \quad \text { with } w(u)=\frac{4}{4+u^{2}} .
$$

Here the sum is a double sum over the imaginary parts of the non-trivial zeros of $\zeta(s)$. He proved in [9] that, as $T \rightarrow \infty$,

$$
F(x, T) \sim \frac{T}{2 \pi} \log x+\frac{T}{2 \pi x^{2}} \log ^{2} T
$$

for $1 \leq x \leq T$ (actually he only proved this for $1 \leq x \leq o(T)$ and the full range was done by Goldston [5]). He conjectured that

$$
F(x, T) \sim \frac{T}{2 \pi} \log T
$$

for $T \leq x \leq T^{M}$, $M$ fixed, which is known as the Strong Pair Correlation Conjecture. From this, one has the (Weak) Pair Correlation Conjecture:

$$
\sum_{\substack{0<\gamma, \gamma^{\prime} \leq T \\ 0<\gamma-\gamma^{\prime} \leq 2 \pi \alpha / \log T}} 1 \sim \frac{T}{2 \pi} \log T \int_{0}^{\alpha}\left[1-\left(\frac{\sin \pi u}{\pi u}\right)^{2}\right] d u,
$$

which draws connections with random matrix theory.

The author studied these further in his thesis [1] (see also [2] and [3]) and derived more precise asymptotic formulas for $F(x, T)$ when $x$ is in various ranges under the Twin Prime Conjecture (TPC) (see Section 4). In the

2000 Mathematics Subject Classification: Primary 11M26. 
present paper, we generalize $F(x, T)$ further to

$$
F_{h}(x, T)=\sum_{0<\gamma, \gamma^{\prime} \leq T} \cos \left(\left(\gamma-\gamma^{\prime}-h\right) \log x\right) w\left(\gamma-\gamma^{\prime}-h\right) .
$$

Note that $F_{h}(x, T)=F_{-h}(x, T)$ and $F_{0}(x, T)=F(x, T)$. This leads to a better understanding of the distribution of larger differences between the zeros. Our main results are the following theorems. Here and throughout the paper, $\widetilde{h}=|h|+1$.

TheOREM 1.1. For $1 \leq x \leq T / \log T$,

$$
\begin{aligned}
F_{h}(x, T)= & \frac{T}{2 \pi}\left[\frac{4 \cos (h \log x)}{4+h^{2}} \log x-\frac{8 h \sin (h \log x)}{\left(4+h^{2}\right)^{2}}\right] \\
& +\frac{T}{2 \pi x^{2}}\left[\left(\log \frac{T}{2 \pi}\right)^{2}-2 \log \frac{T}{2 \pi}\right]+O(x \log x)+O\left(\frac{\widetilde{h} T}{x^{1 / 2-\varepsilon}}\right) .
\end{aligned}
$$

Theorem 1.2. Assume TPC. For $M \geq 3$ and $T / \log ^{M} T \leq x$, $F_{h}(x, T)$

$$
\begin{aligned}
= & \frac{T}{\pi}\left[\frac{2 \cos (h \log x)}{4+h^{2}} \log x-\frac{4 h \sin (h \log x)}{\left(4+h^{2}\right)^{2}}\right] \\
& +\frac{T}{\pi} \int_{1}^{\infty}\left[-\frac{2 \cos (h \log x)}{4+h^{2}} \frac{1}{y}-\frac{4 f(y)}{y^{2}} \cos (h \log x)+G_{1}(y)+G_{2}(y)\right] \\
& \times \frac{\sin \frac{T y}{x}}{\frac{T y}{x}} d y \\
& -\frac{x}{\pi} \int_{0}^{T / x} \frac{\sin u}{u} d u\left[\frac{3 \cos (h \log x)}{9+h^{2}}+\frac{h \sin (h \log x)}{9+h^{2}}\right] \\
& -\frac{x}{\pi} \int_{0}^{T / x} \frac{\sin u}{u} d u\left[\frac{\cos (h \log x)}{1+h^{2}}-\frac{h \sin (h \log x)}{1+h^{2}}\right] \\
& +\frac{T}{\pi} \sum_{k=1}^{\infty} \frac{\mathfrak{S}(k)}{k^{2}} \int_{0}^{1} y \cos \left(h \log \frac{k x}{y}\right) \frac{\sin \frac{T y}{x}}{\frac{T y}{x}} d y \\
& +O\left(\widetilde{h} \frac{x^{1+6 \varepsilon}}{T}\right)+O\left(\widetilde{h} x^{1 / 2+7 \varepsilon}\right)+O\left(\widetilde{h} \frac{x^{2}}{T^{2-2 \varepsilon}}\right)+O\left(\frac{\widetilde{h} T}{\log ^{M-2} T}\right)
\end{aligned}
$$

where $G_{1}(y)$ and $G_{2}(y)$ are defined in Lemma 4.2 . 
TheOrem 1.3. Assume TPC. For $M \geq 3$ and $T / \log ^{M} T \leq x \leq T$,

$$
\begin{aligned}
F_{h}(x, T)= & \frac{T}{\pi}\left[\frac{2 \cos (h \log x)}{4+h^{2}} \log x-\frac{4 h \sin (h \log x)}{\left(4+h^{2}\right)^{2}}\right] \\
& +O(\widetilde{h} x)+O\left(\frac{\widetilde{h} T}{\log ^{M-2} T}\right) .
\end{aligned}
$$

Theorem 1.4. Assume TPC. For $M \geq 3$ and $T \leq x \leq T^{2-29 \varepsilon}$,

$$
\begin{aligned}
F_{h}(x, T)= & \frac{T}{2 \pi} \log \frac{T}{2 \pi e}\left[\frac{4 \cos (h \log x)}{4+h^{2}}\right] \\
& +O\left(\widetilde{h} T\left(\frac{T}{x}\right)^{1 / 2-\varepsilon}\right)+O\left(\frac{\widetilde{h} T}{\log ^{M-2} T}\right) .
\end{aligned}
$$

For real $\alpha$, let $F_{h}(\alpha):=\left(\frac{T}{2 \pi} \log T\right)^{-1} F_{h}\left(T^{\alpha}, T\right)$. Then $F_{h}(\alpha)=F_{h}(-\alpha)$. Based on the above theorems, one may make the following

Conjecture 1.1. For any arbitrarily large $A$ and $h=o\left(\log ^{1 / 3} T\right)$, as $T \rightarrow \infty$,

$$
F_{h}(\alpha)= \begin{cases}(1+o(1)) T^{-2 \alpha} \log T+\alpha \frac{4 \cos (h \log T \alpha)}{4+h^{2}}+o(1) & \text { if } 0 \leq \alpha \leq 1, \\ \frac{4 \cos (h \log T \alpha)}{4+h^{2}}+o(1) & \text { if } 1 \leq \alpha \leq A\end{cases}
$$

By convolving $F_{h}(\alpha)$ with an appropriate kernel $\widehat{r}(\alpha)$,

$$
\begin{aligned}
\left(\frac{T}{2 \pi} \log T\right)^{-1} \sum_{0<\gamma, \gamma^{\prime} \leq T} r\left(\left(\gamma-\gamma^{\prime}-h\right) \frac{\log T}{2 \pi}\right) & w\left(\gamma-\gamma^{\prime}-h\right) \\
& =\int_{-\infty}^{\infty} F_{h}(\alpha) \widehat{r}(\alpha) d \alpha
\end{aligned}
$$

where $\widehat{r}(\alpha)=\int_{-\infty}^{\infty} r(u) e^{-2 \pi i \alpha u} d u$ for even $r(u)$ only. Conjecture 1.1 and (1) lead to

Conjecture 1.2. For fixed $\alpha>0$ and $h=o\left(\log ^{1 / 3} T\right)$,

$$
\begin{aligned}
\left(\frac{T}{2 \pi} \log T\right)^{-1} & \sum_{\substack{0<\gamma \neq \gamma^{\prime} \leq T \\
\left|\gamma-\gamma^{\prime}-h\right| \leq 2 \pi \alpha / \log T}} 1 \\
& \sim \int_{-\alpha+(h \log T) /(2 \pi)}^{\alpha+(h \log T) /(2 \pi)}\left[1-\frac{4}{4+h^{2}}\left(\frac{\sin \pi u}{\pi u}\right)^{2}\right] d u .
\end{aligned}
$$


Conjecture 1.3. For $0<\alpha<\beta \ll \log T$,

$$
\begin{aligned}
\left(\frac{T}{2 \pi} \log T\right)^{-1} & \sum_{\substack{0<\gamma \neq \gamma^{\prime} \leq T \\
2 \pi \alpha / \log T \leq \gamma-\gamma^{\prime} \leq 2 \pi \beta / \log T}} \\
& \sim \int_{\alpha}^{\beta}\left[1-\frac{1}{1+(\pi u / \log T)^{2}}\left(\frac{\sin \pi u}{\pi u}\right)^{2}\right] d u .
\end{aligned}
$$

\section{Some lemmas}

LEMMA 2.1 ([2, Lemma 2.2]). We have, assuming $R H$, for $x \geq 1$,

$$
\begin{aligned}
2 \sum_{\gamma} \frac{x^{i(\gamma-t)}}{1+(t-\gamma)^{2}} & \\
= & -\frac{1}{x} \sum_{n \leq x} \frac{\Lambda(n)}{n^{-1 / 2+i t}}-x \sum_{n>x} \frac{\Lambda(n)}{n^{3 / 2+i t}}+\frac{x^{1 / 2-i t}}{1 / 2+i t}+\frac{x^{1 / 2-i t}}{3 / 2-i t} \\
& +\frac{\log \tau}{x}+\frac{1}{x}\left[\frac{\zeta^{\prime}}{\zeta}\left(\frac{3}{2}-i t\right)-\log 2 \pi\right]+O\left(\frac{1}{x \tau}\right),
\end{aligned}
$$

where the sum is over all the imaginary parts of the zeros of the Riemann zeta function, and $\tau=|t|+2$, and $\Lambda(n)$ is von Mangoldt's lambda function.

Write $(2)$ as $\mathcal{L}(x, t)=\mathcal{R}(x, t)$. Let

$$
P(x, T)=\frac{1}{x} \sum_{n \leq x} \frac{\Lambda(n)}{n^{-1 / 2+i t}}+x \sum_{n>x} \frac{\Lambda(n)}{n^{3 / 2+i t}}-\frac{x^{1 / 2-i t}}{1 / 2+i t}-\frac{x^{1 / 2-i t}}{3 / 2-i t},
$$

$Q(x, T)=\frac{\log \tau}{x}, \quad R(x, T)=\frac{1}{x}\left[\frac{\zeta^{\prime}}{\zeta}\left(\frac{3}{2}-i t\right)-\log 2 \pi\right], \quad S(x, T)=O\left(\frac{1}{x \tau}\right)$.

LEMMA 2.2. For $x \geq 1$,

$$
\begin{aligned}
& \int_{0}^{T}|\mathcal{L}(x, t)+\mathcal{L}(x, t-h)|^{2} d t \\
& \quad=2 \pi F(x, T)+2 \pi F(x, T-h)+4 \pi F_{h}(x, T)+O\left(\log ^{3} T\right)+O\left(h \log ^{2} h\right) .
\end{aligned}
$$

Proof. This follows from page 188 of Montgomery [9] and the fact that $F(x, T) \ll T \log ^{2} T$. 
Lemma 2.3. For $x \geq 1$,

$$
\begin{aligned}
\int_{0}^{T} \mid \mathcal{R}(x, t)+ & \left.\mathcal{R}(x, t-h)\right|^{2} d t \\
= & \int_{0}^{T}|P(x, t)+P(x, t-h)|^{2} d t+\frac{4 T}{x^{2}}\left[\left(\log \frac{T}{2 \pi}\right)^{2}-2 \log \frac{T}{2 \pi}\right. \\
& \left.+\left(\frac{1}{2} \sum_{n=1}^{\infty} \frac{\Lambda^{2}(n)(1+\cos (h \log n))}{n^{3}}+2\right)\right]+O\left(\widetilde{h} \log ^{2} T\right) .
\end{aligned}
$$

Proof. This is similar to the proof of Theorem 3.1 in [2].

Lemma 2.4. For $x \geq 1$,

$$
\begin{aligned}
& 4 \pi F_{h}(x, T) \\
& =\int_{0}^{T}|P(x, t)+P(x, t-h)|^{2} d t-\int_{0}^{T}|P(x, t)|^{2} d t-\int_{0}^{T}|P(x, t-h)|^{2} d t \\
& \quad+\frac{2 T}{x^{2}}\left[\left(\log \frac{T}{2 \pi}\right)^{2}-2 \log \frac{T}{2 \pi}+\left(\sum_{n=1}^{\infty} \frac{\Lambda^{2}(n) \cos (h \log n)}{n^{3}}+2\right)\right] \\
& \quad+O\left(\widetilde{h} \log ^{3} T\right) .
\end{aligned}
$$

Proof. This follows from Lemmas 2.2 and 2.3 as well as their special cases when $h=0$.

Lemma 2.5. For any sequence of complex numbers $\left\{a_{n}\right\}_{n=1}^{\infty}$ satisfying $\sum_{n=1}^{\infty} n\left|a_{n}\right|^{2}<\infty$,

$$
\int_{0}^{T}\left|\sum_{n=1}^{\infty} a_{n} n^{-i t}\right|^{2} d t=\sum_{n=1}^{\infty}\left|a_{n}\right|^{2}(T+O(n)) .
$$

Proof. This is Parseval's identity for Dirichlet series. See [10].

Lemma 2.6. Assuming $R H$ and $x \geq 1$, we have

$$
\begin{aligned}
\sum_{n \leq x} \Lambda^{2}(n) n & =\frac{1}{2} x^{2} \log x-\frac{1}{4} x^{2}+O\left(x^{1 / 2+\varepsilon}\right), \\
\sum_{n>x} \frac{\Lambda^{2}(n)}{n^{3}} & =\frac{1}{2} \frac{\log x}{x^{2}}+\frac{1}{4 x^{2}}+O\left(\frac{1}{x^{5 / 2-\varepsilon}}\right) .
\end{aligned}
$$

Proof. Use partial summation and the prime number theorem.

Lemma 2.7. For any real $a$ and $b$ not both zero,

$$
\begin{aligned}
& \int e^{a x} \sin b x d x=\frac{a}{a^{2}+b^{2}} e^{a x} \sin b x-\frac{b}{a^{2}+b^{2}} e^{a x} \cos b x, \\
& \int e^{a x} \cos b x d x=\frac{a}{a^{2}+b^{2}} e^{a x} \cos b x+\frac{b}{a^{2}+b^{2}} e^{a x} \sin b x,
\end{aligned}
$$




$$
\begin{aligned}
\int x e^{a x} \sin b x d x= & {\left[\frac{a x}{a^{2}+b^{2}}-\frac{a^{2}-b^{2}}{\left(a^{2}+b^{2}\right)^{2}}\right] e^{a x} \sin b x } \\
& -\left[\frac{b x}{a^{2}+b^{2}}-\frac{2 a b}{\left(a^{2}+b^{2}\right)^{2}}\right] e^{a x} \cos b x, \\
\int x e^{a x} \cos b x d x= & {\left[\frac{a x}{a^{2}+b^{2}}-\frac{a^{2}-b^{2}}{\left(a^{2}+b^{2}\right)^{2}}\right] e^{a x} \cos b x } \\
& +\left[\frac{b x}{a^{2}+b^{2}}-\frac{2 a b}{\left(a^{2}+b^{2}\right)^{2}}\right] e^{a x} \sin b x .
\end{aligned}
$$

Proof. One can use the integrals $\int e^{(a+i b) x} d x, \int e^{(a-i b) x} d x, \int x e^{(a+i b) x} d x$ and $\int x e^{(a-i b) x} d x$, which are simple to compute.

LEMMA 2.8. Assuming $R H$ and $x \geq 1$, we have

$$
\begin{aligned}
\frac{1}{x^{2}} \sum_{n \leq x} \Lambda^{2}(n) n \cos (h \log n)= & \frac{2 \cos (h \log x)}{4+h^{2}} \log x+\frac{h^{2}-4}{\left(4+h^{2}\right)^{2}} \cos (h \log x) \\
& +\frac{h \sin (h \log x)}{4+h^{2}} \log x-\frac{4 h}{\left(4+h^{2}\right)^{2}} \sin (h \log x) \\
& +O\left(\frac{\tilde{h}}{x^{1 / 2-\varepsilon}}\right), \\
x^{2} \sum_{n>x} \frac{\Lambda^{2}(n)}{n} \cos (h \log n)= & \frac{2 \cos (h \log x)}{4+h^{2}} \log x-\frac{h^{2}-4}{\left(4+h^{2}\right)^{2}} \cos (h \log x) \\
& -\frac{h \sin (h \log x)}{4+h^{2}} \log x-\frac{4 h}{\left(4+h^{2}\right)^{2}} \sin (h \log x) \\
& +O\left(\frac{\tilde{h}}{x^{1 / 2-\varepsilon}}\right) .
\end{aligned}
$$

Proof. We shall prove the first formula. The other one is very similar. Let $A(x)=x^{-2} \sum_{n \leq x} \Lambda^{2}(n) n$. By partial summation and Lemma 2.6,

$$
\begin{aligned}
\frac{1}{x^{2}} \sum_{n \leq x} \Lambda^{2}(n) n \cos (h \log n) & \\
= & \frac{A(x)}{x^{2}} \cos (h \log x)+\frac{h}{x^{2}} \int_{1}^{x} A(u) \frac{\sin (h \log u)}{u} d u \\
= & {\left[\frac{1}{2} \log x-\frac{1}{4}\right] \cos (h \log x)+\frac{h}{x^{2}} \int_{1}^{x}\left[\frac{1}{2} \log u-\frac{1}{4}\right] u \sin (h \log u) d u } \\
& +O\left(\frac{\widetilde{h}}{x^{1 / 2-\varepsilon}}\right)
\end{aligned}
$$




$$
\begin{aligned}
= & {\left[\frac{1}{2} \log x-\frac{1}{4}\right] \cos (h \log x)+\frac{h}{x^{2}}\left[\frac{1}{2} \int_{0}^{\log x} v e^{2 v} \sin h v d v\right.} \\
& \left.-\frac{1}{4} \int_{0}^{\log x} e^{2 v} \sin h v d v\right]+O\left(\frac{\widetilde{h}}{x^{1 / 2-\varepsilon}}\right),
\end{aligned}
$$

which gives the desired result after applying Lemma 2.7 with $a=2$ and $b=h$, and some algebra.

3. Proof of Theorem 1.1. First, note that

$$
P(x, t)=\frac{1}{x^{1 / 2}}\left[\sum_{n \leq x} \Lambda(n)\left(\frac{x}{n}\right)^{-1 / 2+i t}+\sum_{n>x} \Lambda(n)\left(\frac{x}{n}\right)^{3 / 2+i t}\right]+O\left(\frac{x^{1 / 2}}{\tau}\right) .
$$

Thus,

$$
\begin{aligned}
P(x, t)+P(x, t-h)= & \frac{1}{x^{1 / 2}}\left[\sum_{n \leq x} \Lambda(n)\left(1+n^{i h}\right)\left(\frac{x}{n}\right)^{-1 / 2+i t}\right. \\
& \left.+\sum_{n>x} \Lambda(n)\left(1+n^{i h}\right)\left(\frac{x}{n}\right)^{3 / 2+i t}\right]+O\left(\frac{x^{1 / 2}}{\tau}\right) .
\end{aligned}
$$

So, the first integral in Lemma 2.4 is

$$
\begin{aligned}
\frac{1}{x} \int_{0}^{T} \mid & \sum_{n \leq x} \Lambda(n)\left(1+n^{i h}\right)\left(\frac{x}{n}\right)^{-1 / 2+i t}+\left.\sum_{n>x} \Lambda(n)\left(1+n^{i h}\right)\left(\frac{x}{n}\right)^{3 / 2+i t}\right|^{2} d t \\
& +O\left(\left[\sum_{n \leq x} \Lambda(n)\left(\frac{x}{n}\right)^{-1 / 2}+\sum_{n>x} \Lambda(n)\left(\frac{x}{n}\right)^{3 / 2}\right] \int_{0}^{T} \frac{1}{\tau^{2}} d t\right)+O\left(\int_{0}^{T} \frac{x}{\tau^{4}} d t\right) \\
= & \frac{1}{x} \sum_{n \leq x} \Lambda^{2}(n)\left|1+n^{i h}\right|^{2}\left(\frac{x}{n}\right)^{-1}(T+O(n)) \\
& +\frac{1}{x} \sum_{n>x} \Lambda^{2}(n)\left|1+n^{i h}\right|^{2}\left(\frac{x}{n}\right)^{3}(T+O(n))+O(x) \\
= & \frac{2 T}{x^{2}} \sum_{n \leq x} \Lambda^{2}(n) n(1+\cos (h \log n))+2 T x^{2} \sum_{n>x} \frac{\Lambda^{2}(n)}{n^{3}}(1+\cos (h \log n)) \\
& +O(x \log x) .
\end{aligned}
$$

Similarly (or by setting $h=0$ ), each of the second and third integrals in Lemma 2.4 is

$$
\frac{T}{x^{2}} \sum_{n \leq x} \Lambda^{2}(n) n+T x^{2} \sum_{n>x} \frac{\Lambda^{2}(n)}{n^{3}}+O(x \log x) .
$$


Therefore,

$$
\begin{aligned}
4 \pi F_{h}(x, T)= & 2 T\left[\frac{1}{x^{2}} \sum_{n \leq x} \Lambda^{2}(n) n \cos (h \log n)+x^{2} \sum_{n>x} \frac{\Lambda^{2}(n)}{n^{3}} \cos (h \log n)\right] \\
& +\frac{2 T}{x^{2}}\left[\left(\log \frac{T}{2 \pi}\right)^{2}-2 \log \frac{T}{2 \pi}\right] \\
& +O\left(\frac{T}{x^{2}}\right)+O\left(\widetilde{h} \log ^{3} T\right)+O(x \log x) \\
= & 2 T\left[\frac{4 \cos (h \log x)}{4+h^{2}} \log x-\frac{8 h \sin (h \log x)}{\left(4+h^{2}\right)^{2}}\right] \\
& +\frac{2 T}{x^{2}}\left[\left(\log \frac{T}{2 \pi}\right)^{2}-2 \log \frac{T}{2 \pi}\right]+O(x \log x)+O\left(\frac{\widetilde{h} T}{x^{1 / 2-\varepsilon}}\right)
\end{aligned}
$$

by Lemma 2.8 . The theorem follows after dividing through by $4 \pi$.

4. Twin Prime Conjecture and smooth weight. We shall use a quantitative form of the Twin Prime Conjecture (TPC) as follows: For any $\varepsilon>0$,

$$
\sum_{n=1}^{N} \Lambda(n) \Lambda(n+d)=\mathfrak{S}(d) N+O\left(N^{1 / 2+\varepsilon}\right) \quad \text { uniformly in }|d| \leq N .
$$

Here

$$
\mathfrak{S}(d)= \begin{cases}2 \prod_{p>2}\left(1-\frac{1}{(p-1)^{2}}\right) \prod_{p \mid d, p>2} \frac{p-1}{p-2} & \text { if } d \text { is even } \\ 0 & \text { if } d \text { is odd }\end{cases}
$$

Let $K$ and $M$ be some large positive integers ( $K$ may depend on $\varepsilon$ ). Set $U=\log ^{M} T$ and $\Delta=1 /\left(2^{K} U\right)$. We recall the smooth weight $\Psi_{U}(t)$ in [3] with:

1. support in $[-1 / U, 1+1 / U]$,

2. $0 \leq \Psi_{U}(t) \leq 1$,

3. $\Psi_{U}(t)=1$ for $1 / U \leq t \leq 1-1 / U$,

4. $\Psi_{U}^{(j)}(t) \ll U^{j}$ for $j=1, \ldots, K$.

This weight function satisfies the requirements in Goldston and Gonek [6]. One more thing to note is that

$$
\operatorname{Re} \widehat{\Psi}_{U}(y)=\frac{\sin 2 \pi y}{2 \pi y}\left(\frac{\sin 2 \pi \Delta y}{2 \pi \Delta y}\right)^{K+1}
$$

where $\widehat{f}(y)=\int_{-\infty}^{\infty} f(t) e(y t) d t$. 
We also need to study

$$
\begin{array}{ll}
S_{\alpha}^{h}(y):=\sum_{k \leq y} \mathfrak{S}(k) k^{\alpha} \cos \left(h \log \frac{k x}{y}\right)-\int_{0}^{y} u^{\alpha} \cos \left(h \log \frac{u x}{y}\right) d u & \text { for } \alpha \geq 0, \\
T_{\alpha}^{h}(y):=\sum_{k>y} \frac{\mathfrak{S}(k)}{k^{\alpha}} \cos \left(h \log \frac{k x}{y}\right)-\int_{y}^{\infty} \frac{1}{u^{\alpha}} \cos \left(h \log \frac{u x}{y}\right) d u & \text { for } \alpha>1 .
\end{array}
$$

Then from [4],

$$
S_{0}(y):=S_{0}^{0}(y)=-\frac{1}{2} \log y+O\left((\log y)^{2 / 3}\right)=-\frac{1}{2} \log y+\varepsilon(y) .
$$

By partial summation and Lemma 2.7, for $\alpha>0$,

$$
\begin{aligned}
S_{\alpha}^{h}(y)= & \varepsilon(y) y^{\alpha} \cos (h \log x)-\frac{\alpha \cos (h \log x)}{2\left(\alpha^{2}+h^{2}\right)} y^{\alpha}-\frac{h \sin (h \log x)}{2\left(\alpha^{2}+h^{2}\right)} y^{\alpha} \\
& -\int_{0}^{y} \varepsilon(u) u^{\alpha-1}\left[\alpha \cos \left(h \log \frac{u x}{y}\right)-h \sin \left(h \log \frac{u x}{y}\right)\right] d u,
\end{aligned}
$$

and, for $\alpha>1$,

$$
\begin{aligned}
T_{\alpha}^{h}(y)= & -\frac{\varepsilon(y)}{y^{\alpha}} \cos (h \log x)-\frac{\alpha \cos (h \log x)}{2\left(\alpha^{2}+h^{2}\right)} \frac{1}{y^{\alpha}}+\frac{h \sin (h \log x)}{2\left(\alpha^{2}+h^{2}\right)} \frac{1}{y^{\alpha}} \\
& +\int_{y}^{\infty} \frac{\varepsilon(u)}{u^{\alpha+1}}\left[\alpha \cos \left(h \log \frac{u x}{y}\right)+h \sin \left(h \log \frac{u x}{y}\right)\right] d u .
\end{aligned}
$$

Let

$$
f(y):=\int_{0}^{y}\left(\varepsilon(u)-\frac{B}{2}\right) d u
$$

where $B=-C_{0}-\log 2 \pi$ and $C_{0}$ is Euler's constant. Note that

$$
f(y) \ll y^{1 / 2+\varepsilon}
$$

(see Lemma 2.2 of [3]). From (4) and (5),

$$
\begin{aligned}
& S_{2}^{h}(y) \frac{1}{y^{3}}+T_{2}^{h}(y) y \\
= & -\frac{2 \cos (h \log x)}{4+h^{2}} \frac{1}{y}-\frac{1}{y^{3}} \int_{0}^{y} u \varepsilon(u)\left[2 \cos \left(h \log \frac{u x}{y}\right)-h \sin \left(h \log \frac{u x}{y}\right)\right] d u \\
& +y \int_{y}^{\infty} \frac{\varepsilon(u)}{u^{3}}\left[2 \cos \left(h \log \frac{u x}{y}\right)+h \sin \left(h \log \frac{u x}{y}\right)\right] d u
\end{aligned}
$$

LEMMA 4.1. We have

$$
I+J=-\frac{1}{y^{3}} \int_{0}^{y} u \varepsilon(u)\left[2 \cos \left(h \log \frac{u x}{y}\right)-h \sin \left(h \log \frac{u x}{y}\right)\right] d u
$$




$$
\begin{aligned}
& +y \int_{y}^{\infty} \frac{\varepsilon(u)}{u^{3}}\left[2 \cos \left(h \log \frac{u x}{y}\right)+h \sin \left(h \log \frac{u x}{y}\right)\right] d u \\
= & -\frac{4 f(y)}{y} \cos (h \log x) \\
& +\frac{1}{y^{3}} \int_{0}^{y} f(u)\left[\left(2-h^{2}\right) \cos \left(h \log \frac{u x}{y}\right)-3 h \sin \left(h \log \frac{u x}{y}\right)\right] d u \\
& +y \int_{y}^{\infty} \frac{f(u)}{u^{4}}\left[\left(6-h^{2}\right) \cos \left(h \log \frac{u x}{y}\right)+5 h \sin \left(h \log \frac{u x}{y}\right)\right] d u .
\end{aligned}
$$

Proof. I can be rewritten as

$$
\begin{aligned}
& -\frac{1}{y^{3}} \int_{0}^{y} u\left(\varepsilon(u)-\frac{B}{2}\right)\left[2 \cos \left(h \log \frac{u x}{y}\right)-h \sin \left(h \log \frac{u x}{y}\right)\right] d u \\
& -\frac{B}{2} \frac{1}{y^{3}} \int_{0}^{y} u\left[2 \cos \left(h \log \frac{u x}{y}\right)-h \sin \left(h \log \frac{u x}{y}\right)\right] d u=-I_{1}-I_{2} .
\end{aligned}
$$

By a substitution $v=\log \frac{u x}{y}$ and Lemma 2.7,

$$
I_{2}=\frac{B}{2} \frac{1}{y} \cos (h \log x)
$$

By integration by parts and (6),

(9) $I_{1}=\frac{f(y)}{y^{2}}[2 \cos (h \log x)-h \sin (h \log x)]$

$$
-\frac{1}{y^{3}} \int_{0}^{y} f(u)\left[\left(2-h^{2}\right) \cos \left(h \log \frac{u x}{y}\right)-3 h \sin \left(h \log \frac{u x}{y}\right)\right] d u \text {. }
$$

Similarly, $J$ can be rewritten as

$$
\begin{aligned}
y \int_{y}^{\infty} \frac{\varepsilon(u)-B / 2}{u^{3}}\left[2 \cos \left(h \log \frac{u x}{y}\right)+h \sin \left(h \log \frac{u x}{y}\right)\right] d u \\
+\frac{B}{2} y \int_{y}^{\infty} \frac{1}{u^{3}}\left[2 \cos \left(h \log \frac{u x}{y}\right)+h \sin \left(h \log \frac{u x}{y}\right)\right] d u=J_{1}+J_{2} .
\end{aligned}
$$

By a substitution $v=\log \frac{u x}{y}$ and Lemma 2.7,

$$
J_{2}=\frac{B}{2} \frac{1}{y} \cos (h \log x) \text {. }
$$


By integration by parts and (6),

$$
\begin{aligned}
J_{1}= & -\frac{f(y)}{y^{2}}[2 \cos (h \log x)+h \sin (h \log x)] \\
& +y \int_{y}^{\infty} \frac{f(u)}{u^{4}}\left[\left(6-h^{2}\right) \cos \left(h \log \frac{u x}{y}\right)+5 h \sin \left(h \log \frac{u x}{y}\right)\right] d u .
\end{aligned}
$$

Equations (8)-(11) together give the lemma.

LEMMA 4.2. We have

$S_{2}^{h}(y) \frac{1}{y^{3}}+T_{2}^{h}(y) y=-\frac{2 \cos (h \log x)}{4+h^{2}} \frac{1}{y}-\frac{4 f(y)}{y^{2}} \cos (h \log x)+G_{1}(y)+G_{2}(y)$ where

$$
\begin{aligned}
& G_{1}(y)=\frac{1}{y^{3}} \int_{0}^{y} f(u)\left[\left(2-h^{2}\right) \cos \left(h \log \frac{u x}{y}\right)-3 h \sin \left(h \log \frac{u x}{y}\right)\right] d u, \\
& G_{2}(y)=y \int_{y}^{\infty} \frac{f(u)}{u^{4}}\left[\left(6-h^{2}\right) \cos \left(h \log \frac{u x}{y}\right)+5 h \sin \left(h \log \frac{u x}{y}\right)\right] d u .
\end{aligned}
$$

Proof. Combine (7) and Lemma 4.1.

LEMMA 4.3 ([3, Lemma 3.3]). For any integer $n \geq 1$, we have

$$
\int_{1}^{\infty} \frac{1}{y^{n}} \operatorname{Re} \widehat{\Psi}_{U}\left(\frac{T y}{2 \pi x}\right) d y=\int_{1}^{\infty} \frac{1}{y^{n}} \frac{\sin \frac{T}{x} y}{\frac{T}{x} y} d y+O\left(\Delta \log \frac{1}{\Delta}\right) .
$$

When $n \neq 2$, the error term can be replaced by $O(\Delta)$.

LEMMA 4.4 ([3, Lemma 3.4]). If $F(y) \ll y^{-3 / 2+\varepsilon}$ for $y \geq 1$, then

$$
\int_{1}^{\infty} F(y) \operatorname{Re} \widehat{\Psi}_{U}\left(\frac{T y}{2 \pi x}\right) d y=\int_{1}^{\infty} F(y) \frac{\sin \frac{T}{x} y}{\frac{T}{x} y} d y+O(\Delta) .
$$

5. Proof of Theorem 1.2. Throughout this section, we assume $\tau=$ $T^{1-\varepsilon} \leq T / \log ^{M} T \leq x \leq T^{2-2 \varepsilon}, U=\log ^{M} T$ for $M>2, H^{*}=\tau^{-2} x^{2 /(1-\varepsilon)}$, and $\Psi_{U}(t)$ is defined as in the previous section. The implicit constants in the error terms may depend on $\varepsilon, K$ and $M$.

Our method is that of Goldston and Gonek [6] and it is very similar to [3]. Let $s=\sigma+i t$,

$$
\begin{aligned}
A_{h}(s) & :=\sum_{n \leq x} \frac{\Lambda(n)\left(1+n^{i h}\right)}{n^{s}}, & A_{h}^{*}(s):=\sum_{n>x} \frac{\Lambda(n)\left(1+n^{i h}\right)}{n^{s}}, \\
A(s) & :=\frac{1}{2} A_{0}(s), & A^{*}(s):=\frac{1}{2} A_{0}^{*}(s) .
\end{aligned}
$$


By Lemma 2.4, with slight modifications, one has

$$
\begin{aligned}
4 \pi F_{h}(x, T)= & \int_{0}^{T} \mid \frac{1}{x}\left(A_{h}\left(-\frac{1}{2}+i t\right)-\int_{1}^{x}\left(1+u^{i h}\right) u^{1 / 2-i t} d u\right) \\
& +\left.x\left(A_{h}^{*}\left(\frac{3}{2}+i t\right)-\int_{x}^{\infty}\left(1+u^{i h}\right) u^{-3 / 2-i t} d u\right)\right|^{2} d t \\
& -2 \int_{0}^{T} \mid \frac{1}{x}\left(A\left(-\frac{1}{2}+i t\right)-\int_{1}^{x} u^{1 / 2-i t} d u\right) \\
& +\left.x\left(A^{*}\left(\frac{3}{2}+i t\right)-\int_{x}^{\infty} u^{-3 / 2-i t} d u\right)\right|^{2} d t+O\left(\widetilde{h} \log ^{3} T\right) .
\end{aligned}
$$

Inserting $\Psi_{U}(t / T)$ into the integral and extending the range of integration to the whole real line, we get

$$
\begin{aligned}
4 \pi F(x, T)= & \frac{1}{x^{2}} I_{1}(x, T)+x^{2} I_{2}(x, T)-\frac{2}{x^{2}} I_{3}(x, T)-2 x^{2} I_{4}(x, T) \\
& +O\left(\frac{T(\log T)^{2}}{U}\right)+O\left(\frac{x^{1+6 \varepsilon}}{T}\right)
\end{aligned}
$$

where

$$
\begin{aligned}
& I_{1}(x, T)=\int_{-\infty}^{\infty}\left|A_{h}\left(-\frac{1}{2}+i t\right)-\int_{1}^{x}\left(1+u^{i h}\right) u^{1 / 2-i t} d u\right|^{2} \Psi_{U}\left(\frac{t}{T}\right) d t \\
& I_{2}(x, T)=\int_{-\infty}^{\infty}\left|A_{h}^{*}\left(\frac{3}{2}+i t\right)-\int_{x}^{\infty}\left(1+u^{i h}\right) u^{-3 / 2-i t} d u\right|^{2} \Psi_{U}\left(\frac{t}{T}\right) d t \\
& I_{3}(x, T)=\int_{-\infty}^{\infty}\left|A\left(-\frac{1}{2}+i t\right)-\int_{1}^{x} u^{1 / 2-i t} d u\right|^{2} \Psi_{U}\left(\frac{t}{T}\right) d t \\
& I_{4}(x, T)=\int_{-\infty}^{\infty}\left|A^{*}\left(\frac{3}{2}+i t\right)-\int_{x}^{\infty} u^{-3 / 2-i t} d u\right|^{2} \Psi_{U}\left(\frac{t}{T}\right) d t
\end{aligned}
$$

by Lemma 1 of [7] with modification $V=-T / U$ and $T-T / U$, and $W=$ $2 T / U$. The contributions from the cross terms are estimated via Theorem 3 of [6]. Note that by partial summation with the Riemann Hypothesis and TPC,

$$
\begin{gathered}
\sum_{n \leq x} \Lambda(n)\left(1+n^{i h}\right)=\int_{1}^{x}\left(1+u^{i h}\right) d u+O\left(\widetilde{h} x^{1 / 2+\varepsilon}\right) \\
\sum_{n \leq x} \Lambda(n) \Lambda(n+k)\left(1+n^{i h}\right)\left(1+(n+k)^{-i h}\right) \\
=\mathfrak{S}(k) \int_{1}^{x}\left(1+u^{i h}\right)(1+(u+k))^{-i h} d u+O\left(\widetilde{h} x^{1 / 2+\varepsilon}\right) .
\end{gathered}
$$


By Corollary 1 of [6] (see also the calculations at the end of [6] and [7]),

$$
\begin{aligned}
I_{1}(x, T)= & \widehat{\Psi}_{U}(0) T \sum_{n \leq x} \Lambda^{2}(n) n\left|1+n^{i h}\right|^{2} \\
& +4 \pi\left(\frac{T}{2 \pi}\right)^{3} \int_{T / 2 \pi x}^{\infty}\left[\sum_{k \leq 2 \pi x v / T} \mathfrak{S}(k) k^{2}\left(1+\left(\frac{k T}{2 \pi v}\right)^{i h}\right)\right. \\
& \left.\times\left(1+\left(\frac{k T}{2 \pi v}+k\right)^{-i h}\right)\right] \operatorname{Re} \widehat{\Psi}_{U}(v) \frac{d v}{v^{3}} \\
& -4 \pi\left(\frac{T}{2 \pi}\right)^{3} \int_{T / 2 \pi \tau x}^{\infty}\left[\int_{0}^{2 \pi x v / T} u^{2}\left|1+\left(\frac{u T}{2 \pi v}\right)^{i h}\right|^{2} d u\right] \operatorname{Re} \widehat{\Psi}_{U}(v) \frac{d v}{v^{3}} \\
& +O\left(\widetilde{h} \frac{x^{3+6 \varepsilon}}{T}\right)+O\left(\widetilde{h} x^{5 / 2+7 \varepsilon}\right) .
\end{aligned}
$$

Note that

$$
\begin{aligned}
& \left(1+\left(\frac{k T}{2 \pi v}\right)^{i h}\right)\left(1+\left(\frac{k T}{2 \pi v}+k\right)^{-i h}\right) \\
= & \left|1+\left(\frac{k T}{2 \pi v}\right)^{i h}\right|^{2}+\left(1+\left(\frac{k T}{2 \pi v}\right)^{i h}\right)\left(\left(\frac{k T}{2 \pi v}+k\right)^{-i h}-\left(\frac{k T}{2 \pi v}\right)^{-i h}\right) \\
= & \left|1+\left(\frac{k T}{2 \pi v}\right)^{i h}\right|^{2}+\left(1+\left(\frac{k T}{2 \pi v}\right)^{i h}\right)\left(\frac{k T}{2 \pi v}\right)^{-i h}\left(\left(1+\frac{2 \pi v}{T}\right)^{-i h}-1\right) \\
= & \left|1+\left(\frac{k T}{2 \pi v}\right)^{i h}\right|^{2}+O\left(\min \left(\frac{h v}{T}, 1\right)\right) .
\end{aligned}
$$

Thus,

$$
\begin{aligned}
\int_{T / 2 \pi x}^{\infty}\left[\sum_{k \leq 2 \pi x v / T} \mathfrak{S}(k) k^{2}\left(1+\left(\frac{k T}{2 \pi v}\right)^{i h}\right)\left(1+\left(\frac{k T}{2 \pi v}+k\right)^{-i h}\right)\right] \operatorname{Re} \widehat{\Psi}_{U}(v) \frac{d v}{v^{3}} \\
=\int_{T / 2 \pi x}^{\infty}\left[\sum_{k \leq 2 \pi x v / T} \mathfrak{S}(k) k^{2}\left|1+\left(\frac{k T}{2 \pi v}\right)^{i h}\right|^{2}\right] \operatorname{Re} \widehat{\Psi}_{U}(v) \frac{d v}{v^{3}} \\
+O\left(\int_{T / 2 \pi x}^{T^{2-\varepsilon} / x}\left(\frac{x v}{T}\right)^{3} \frac{h v}{T} \frac{1}{v} \frac{d v}{v^{3}}+\int_{T^{2-\varepsilon} / x}^{\infty}\left(\frac{x v}{T}\right)^{3} \frac{1}{\Delta v^{2}} \frac{d v}{v^{3}}\right) \\
=\int_{T / 2 \pi x}^{\infty}\left[\sum_{k \leq 2 \pi x v / T} \mathfrak{S}(k) k^{2}\left|1+\left(\frac{k T}{2 \pi v}\right)^{i h}\right|^{2}\right] \operatorname{Re} \widehat{\Psi}_{U}(v) \frac{d v}{v^{3}} \\
+O\left(\frac{h x^{2}}{T^{2+\varepsilon}}+\frac{x^{4}}{\Delta T^{5-\varepsilon}}\right)
\end{aligned}
$$


as $\sum_{k \leq x} \mathfrak{S}(k) \sim x$ and $\operatorname{Re} \widehat{\Psi}_{U}(v) \ll \min \left(1 / v, 1 / \Delta v^{2}\right)$. Therefore, $I_{1}(x, T)$

$$
\begin{aligned}
= & T \sum_{n \leq x} \Lambda^{2}(n) n(2+2 \cos (h \log n)) \\
& +4 \pi\left(\frac{T}{2 \pi}\right)^{3} \int_{T / 2 \pi x}^{\infty}\left[\sum_{k \leq 2 \pi x v / T} \mathfrak{S}(k) k^{2}\left(2+2 \cos \left(h \log \frac{k T}{2 \pi v}\right)\right)\right. \\
& \left.-\int_{0}^{2 \pi x v / T} u^{2}\left(2+2 \cos \left(h \log \frac{u T}{2 \pi v}\right)\right) d u\right] \operatorname{Re} \widehat{\Psi}_{U}(v) \frac{d v}{v^{3}} \\
& -4 \pi\left(\frac{T}{2 \pi}\right)^{3} \int_{0}^{T / 2 \pi x} \int_{0}^{2 \pi x v / T} u^{2}\left(2+2 \cos \left(h \log \frac{u T}{2 \pi v}\right)\right) d u \operatorname{Re} \widehat{\Psi}_{U}(v) \frac{d v}{v^{3}} \\
& +O\left(\frac{\widetilde{h} x^{3+6 \varepsilon}}{T}\right)+O\left(\widetilde{h} x^{5 / 2+7 \varepsilon}\right)+O\left(\widetilde{h} x^{2} T^{1-\varepsilon}\right)+O\left(\frac{x^{4}}{\Delta T^{2-\varepsilon}}\right) .
\end{aligned}
$$

Similarly, by Corollary 2 of [6],

$$
\begin{aligned}
I_{2}(x, T)= & T \sum_{x<n} \frac{\Lambda^{2}(n)}{n^{3}}(2+2 \cos (h \log n)) \\
& +\frac{8 \pi^{2}}{T} \int_{0}^{T H^{*} / 2 \pi x}\left[\sum_{2 \pi x v / T \leq k \leq H^{*}} \frac{\mathfrak{S}(k)}{k^{2}}\left(2+2 \cos \left(h \log \frac{k T}{2 \pi v}\right)\right)\right. \\
& \left.-\int_{2 \pi x v / T}^{H^{*}} \frac{1}{u^{2}}\left(2+2 \cos \left(h \log \frac{u T}{2 \pi v}\right)\right) d u\right] \operatorname{Re} \widehat{\Psi}_{U}(v) v d v \\
& +O\left(\widetilde{h} T^{-1} x^{-1+6 \varepsilon}\right)+O\left(\widetilde{h} x^{-3 / 2+7 \varepsilon}\right) \\
& +O\left(\widetilde{h} T^{1-\varepsilon / 2} x^{-2}\right)+O\left(\frac{\widetilde{h} H^{*}}{\Delta x^{2}}\right)
\end{aligned}
$$

where the last error term comes from the error term in $(13) . I_{3}(x, T)$ and $I_{4}(x, T)$ are computed in [3] or one can simply set $h=0$ in $I_{1}(x, T)$ and $I_{2}(x, T)$, and divide by 4 . Putting these into (12) with a substitution $y=$ $2 \pi x v / T$ and using Lemma 2.8 , we get

$$
\begin{aligned}
& 4 \pi F_{h}(x, T)=2 T\left[\frac{4 \cos (h \log x)}{4+h^{2}} \log x-\frac{8 h \sin (h \log x)}{\left(4+h^{2}\right)^{2}}\right] \\
& +4 T \int_{1}^{\infty}\left[\sum_{k \leq y} \mathfrak{S}(k) k^{2} \cos \left(h \log \frac{k x}{y}\right)-\int_{0}^{y} u^{2} \cos \left(h \log \frac{u x}{y}\right) d u\right] \operatorname{Re} \widehat{\Psi}_{U}\left(\frac{T y}{2 \pi x}\right) \frac{d y}{y^{3}}
\end{aligned}
$$




$$
\begin{aligned}
& -4 T \int_{0}^{1} \int_{0}^{y} u^{2} \cos \left(h \log \frac{u x}{y}\right) d u \operatorname{Re} \widehat{\Psi}_{U}\left(\frac{T y}{2 \pi x}\right) \frac{d y}{y^{3}} \\
& +4 T \int_{1}^{H^{*}}\left[\sum_{y \leq k \leq H^{*}} \frac{\mathfrak{S}(k) \cos \left(h \log \frac{k x}{y}\right)}{k^{2}}-\int_{y}^{H^{*}} \frac{\cos \left(h \log \frac{u x}{y}\right)}{u^{2}} d u\right] \\
& \times \operatorname{Re} \widehat{\Psi}_{U}\left(\frac{T y}{2 \pi x}\right) y d y \\
& +4 T \int_{0}^{1}\left[\sum_{k \leq H^{*}} \frac{\mathfrak{S}(k) \cos \left(h \log \frac{k x}{y}\right)}{k^{2}}-\int_{y}^{H^{*}} \frac{\cos \left(h \log \frac{u x}{y}\right)}{u^{2}} d u\right] \\
& \times \operatorname{Re} \widehat{\Psi}_{U}\left(\frac{T y}{2 \pi x}\right) y d y \\
& +O\left(\frac{\widetilde{h} x^{1+6 \varepsilon}}{T}\right)+O\left(\widetilde{h} x^{1 / 2+7 \varepsilon}\right)+O\left(\frac{\widetilde{h} x^{2}}{T^{2-2 \varepsilon}}\right)+O\left(\frac{\tilde{h} T}{\log ^{M-2} T}\right) .
\end{aligned}
$$

From Lemma 2.7,

$$
\int e^{a x} \cos b x d x=\frac{a}{a^{2}+b^{2}} e^{a x} \cos b x+\frac{b}{a^{2}+b^{2}} e^{a x} \sin b x .
$$

Also,

$$
\begin{aligned}
\int_{0}^{1} \operatorname{Re} \widehat{\Psi}_{U}\left(\frac{T y}{2 \pi x}\right) d y & =\frac{x}{T} \int_{0}^{T / x} \frac{\sin u}{u}\left(1+O\left(\Delta^{2} u^{2}\right)\right) d u \\
& =\frac{x}{T} \int_{0}^{T / x} \frac{\sin u}{u} d u+O\left(\frac{\Delta^{2} T}{x}\right)
\end{aligned}
$$

Using integration by parts, (14) and (15) with an appropriate change of variables, we have

$$
\begin{aligned}
\int_{0}^{1} \int_{0}^{y} u^{2} \cos \left(h \log \frac{u x}{y}\right) & d u \operatorname{Re} \widehat{\Psi}_{U}\left(\frac{T y}{2 \pi x}\right) \frac{d y}{y^{3}} \\
= & \frac{x}{T} \int_{0}^{T / x} \frac{\sin u}{u} d u\left[\frac{3}{9+h^{2}} \cos (h \log x)+\frac{h}{9+h^{2}} \sin (h \log x)\right] \\
& -\int_{0}^{1} \frac{\cos (h \log x)}{y} \int_{0}^{y} \operatorname{Re} \widehat{\Psi}_{U}\left(\frac{T v}{2 \pi x}\right) d v d y+O\left(\frac{\Delta^{2} T}{x}\right)
\end{aligned}
$$

and 


$$
\begin{aligned}
\int_{0}^{1}\left[\sum_{k \leq H^{*}} \frac{\mathfrak{S}(k) \cos \left(h \log \frac{k x}{y}\right)}{k^{2}}-\int_{y}^{H^{*}} \frac{\cos \left(h \log \frac{u x}{y}\right)}{u^{2}} d u\right] \operatorname{Re} \widehat{\Psi}_{U}\left(\frac{T y}{2 \pi x}\right) y d y \\
=\sum_{k=1}^{\infty} \frac{\mathfrak{S}(k)}{k^{2}} \int_{0}^{1} y \cos \left(h \log \frac{k x}{y}\right) \frac{\sin \frac{T y}{x}}{\frac{T y}{x}} d y+O\left(\frac{1}{H^{*}}\right)+O\left(\frac{\Delta^{2} T}{x}\right) \\
-\frac{x}{T} \int_{0}^{T / x} \frac{\sin u}{u} d u\left[\frac{1}{1+h^{2}} \cos (h \log x)-\frac{h}{1+h^{2}} \sin (h \log x)\right] \\
-\int_{0}^{1} \frac{\cos (h \log x)}{y} \int_{0}^{y} \operatorname{Re} \widehat{\Psi}_{U}\left(\frac{T v}{2 \pi x}\right) d v d y+O\left(\frac{\Delta^{2} T}{x}\right) .
\end{aligned}
$$

Therefore, with the notation $S_{\alpha}^{h}(y)$ and $T_{\alpha}^{h}(y)$,

$$
\begin{aligned}
4 \pi F_{h}(x, T)= & 2 T\left[\frac{4 \cos (h \log x)}{4+h^{2}} \log x-\frac{8 h \sin (h \log x)}{\left(4+h^{2}\right)^{2}}\right] \\
& +4 T \int_{1}^{\infty} S_{2}^{h}(y) \operatorname{Re} \widehat{\Psi}_{U}\left(\frac{T y}{2 \pi x}\right) \frac{d y}{y^{3}} \\
& +4 T \int_{1}^{H^{*}}\left(T_{2}^{h}(y)-T_{2}^{h}\left(H^{*}\right)\right) \operatorname{Re} \widehat{\Psi}_{U}\left(\frac{T y}{2 \pi x}\right) y d y \\
& -4 x \int_{0}^{T / x} \frac{\sin u}{u} d u\left[\frac{3 \cos (h \log x)}{9+h^{2}}+\frac{h \sin (h \log x)}{9+h^{2}}\right] \\
& -4 x \int_{0}^{T / x} \frac{\sin u}{u} d u\left[\frac{\cos (h \log x)}{1+h^{2}}-\frac{h \sin (h \log x)}{1+h^{2}}\right] \\
& \left.+4 T \sum_{k=1}^{\infty} \frac{\mathfrak{S}(k)}{k^{2}} \int_{0}^{1} y \cos \left(h \log \frac{k x}{y}\right) \frac{\sin \frac{T y}{x} d y+O\left(\frac{T y}{x} d x^{1+6 \varepsilon}\right.}{T}\right) \\
& +O\left(\widetilde{h} x^{1 / 2+7 \varepsilon}\right)+O\left(\frac{\widetilde{h} x^{2}}{T^{2-2 \varepsilon}}\right)+O\left(\frac{\widetilde{h}^{M} T}{\log ^{M-2} T}\right) .
\end{aligned}
$$

By (3) and (5), $T_{2}^{h}\left(H^{*}\right) \ll h\left(\log H^{*}\right)^{2 / 3} /\left(H^{*}\right)^{2}$. It follows that the contribution from $T_{2}^{h}\left(H^{*}\right)$ in the second integral is $O\left(h T^{-\varepsilon}\right)$. Also, one can extend the upper limit of the second integral to $\infty$ with an error $O\left(h T^{-\varepsilon}\right)$ by (3) and (5) again. Finally, we obtain the theorem by applying Lemmas 4.2-4.4, (6) and dividing by $4 \pi$.

\section{Proof of Theorems 1.3 and 1.4}

Proof of Theorem 1.3. This follows directly from Theorem 1.2 by observing that all the main terms except the first one are $O(x)$ because of $(6)$. 
Before proving Theorem 1.4, we need the following lemmas.

LEMMA 6.1. We have

$\int_{1}^{\infty} \frac{\sin a x}{x^{2 n}} d x$

$$
=\frac{a^{2 n-1}}{(2 n-1) !}\left[\sum_{k=1}^{2 n-1} \frac{(2 n-k-1) !}{a^{2 n-k}} \sin \left(a+(k-1) \frac{\pi}{2}\right)+(-1)^{n} \operatorname{ci}(a)\right]
$$

where

$$
\operatorname{ci}(x)=-\int_{x}^{\infty} \frac{\cos t}{t} d t=C_{0}+\log x+\int_{0}^{x} \frac{\cos t-1}{t} d t
$$

and $C_{0}$ is Euler's constant.

Proof. This is formula 3.761(3) on p. 430 of [8], which can be proved by integration by parts repeatedly.

Lemma 6.2 ([3, Lemma 5.2]). If $F(y) \ll y^{-3 / 2+\varepsilon}$ for $y \geq 1$, then for $T \leq x$,

$$
\int_{1}^{\infty} F(y) \frac{\sin \frac{T}{x} y}{\frac{T}{x} y} d y=\int_{1}^{\infty} F(y) d y+O\left(\left(\frac{T}{x}\right)^{1 / 2-\varepsilon}\right) .
$$

Lemma 6.3. We have

$$
\begin{aligned}
I= & \int_{1}^{\infty} \frac{1}{y^{3}} \int_{0}^{y} f(u)\left[\left(2-h^{2}\right) \cos \left(h \log \frac{u x}{y}\right)-3 h \sin \left(h \log \frac{u x}{y}\right)\right] d u d y \\
= & \int_{0}^{1} f(u)[\cos (h \log u x)-h \sin (h \log u x)] d u \\
& +\int_{1}^{\infty} \frac{f(u)}{u^{2}} d u[\cos (h \log x)-h \sin (h \log x)] .
\end{aligned}
$$

Proof. Because of (6), we can change the order of integration:

$$
\begin{aligned}
I= & \int_{0}^{1} f(u) \int_{1}^{\infty} \frac{1}{y^{3}}\left[\left(2-h^{2}\right) \cos \left(h \log \frac{u x}{y}\right)-3 h \sin \left(h \log \frac{u x}{y}\right)\right] d y d u \\
& +\int_{1}^{\infty} f(u) \int_{u}^{\infty} \frac{1}{y^{3}}\left[\left(2-h^{2}\right) \cos \left(h \log \frac{u x}{y}\right)-3 h \sin \left(h \log \frac{u x}{y}\right)\right] d y d u \\
= & \int_{0}^{1} f(u)\left\{\left(2-h^{2}\right)\left[\frac{2}{4+h^{2}} \cos (h \log u x)+\frac{h}{4+h^{2}} \sin (h \log u x)\right]\right. \\
& \left.-3 h\left[\frac{2}{4+h^{2}} \sin (h \log u x)-\frac{h}{4+h^{2}} \cos (h \log u x)\right]\right\} d u
\end{aligned}
$$




$$
\begin{aligned}
& +\int_{1}^{\infty} \frac{f(u)}{u^{2}}\left\{\left(2-h^{2}\right)\left[\frac{2}{4+h^{2}} \cos (h \log x)+\frac{h}{4+h^{2}} \sin (h \log x)\right]\right. \\
& \left.-3 h\left[\frac{2}{4+h^{2}} \sin (h \log x)-\frac{h}{4+h^{2}} \cos (h \log x)\right]\right\} d u,
\end{aligned}
$$

by substituting $v=\log \frac{u x}{y}$ and applying Lemma 2.7. Now the result follows after some simple algebra.

LEMMA 6.4. We have

$$
\begin{aligned}
J= & \int_{1}^{\infty} y \int_{y}^{\infty} \frac{f(u)}{u^{4}}\left[\left(6-h^{2}\right) \cos \left(h \log \frac{u x}{y}\right)+5 h \sin \left(h \log \frac{u x}{y}\right)\right] d u d y \\
= & -\int_{1}^{\infty} \frac{f(u)}{u^{4}}[3 \cos (h \log u x)+h \sin (h \log u x)] d u \\
& +\int_{1}^{\infty} \frac{f(u)}{u^{2}} d u[3 \cos (h \log x)+h \sin (h \log x)] .
\end{aligned}
$$

Proof. Again, because of (6), we can change the order of integration:

$$
\begin{aligned}
J= & \int_{1}^{\infty} \int_{1}^{u} y\left[\left(6-h^{2}\right) \cos \left(h \log \frac{u x}{y}\right)+5 h \sin \left(h \log \frac{u x}{y}\right)\right] d y d u \\
= & \int_{1}^{\infty} \frac{f(u)}{u^{4}}\left\{\left(6-h^{2}\right)\left[\frac{-2}{4+h^{2}} \cos (h \log u x)+\frac{h}{4+h^{2}} \sin (h \log u x)\right]\right. \\
& +5 h\left[\frac{-2}{4+h^{2}} \sin (h \log u x)-\frac{h}{4+h^{2}} \cos (h \log u x)\right] \\
& -\left(6-h^{2}\right)\left[\frac{-2}{4+h^{2}} \cos (h \log x)+\frac{h}{4+h^{2}} \sin (h \log x)\right] \\
& \left.-5 h\left[\frac{-2}{4+h^{2}} \sin (h \log x)-\frac{h}{4+h^{2}} \cos (h \log x)\right]\right\} d u
\end{aligned}
$$

by substituting $v=\log \frac{u x}{y}$ and applying Lemma 2.7. The result now follows after some simple algebra.

LEMMA 6.5. We have

$$
\begin{aligned}
S= & \sum_{k=1}^{\infty} \frac{\mathfrak{S}(k)}{k^{2}} \int_{0}^{1} y \cos \left(h \log \frac{k x}{y}\right) d y \\
= & {\left[\frac{1}{1+h^{2}} \cos (h \log x)-\frac{h}{1+h^{2}} \sin (h \log x)\right] } \\
& -\left[\frac{4-h^{2}}{2\left(4+h^{2}\right)^{2}} \cos (h \log x)-\frac{2 h}{\left(4+h^{2}\right)^{2}} \sin (h \log x)\right]
\end{aligned}
$$


$+\frac{B}{2}\left[\frac{2}{4+h^{2}} \cos (h \log x)-\frac{h}{4+h^{2}} \sin (h \log x)\right]+\left(1+\frac{B}{2}\right) \cos (h \log x)$

$+\int_{1}^{\infty} \frac{f(u)}{u^{4}}[3 \cos (h \log u x)+h \sin (h \log u x)] d u$.

Proof. By substituting $v=\log \frac{k x}{y}$ and using Lemma 2.7,

$$
S=\frac{2}{4+h^{2}} \sum_{k=1}^{\infty} \frac{\mathfrak{S}(k)}{k^{2}} \cos (h \log k x)-\frac{h}{4+h^{2}} \sum_{k=1}^{\infty} \frac{\mathfrak{S}(k)}{k^{2}} \sin (h \log k x) .
$$

Recall the definition of $S_{0}(u)$ from (3) and use partial summation to obtain

$$
\begin{aligned}
S= & \frac{2}{4+h^{2}} \int_{1}^{\infty} \frac{S_{0}(u)+u}{u^{3}}[2 \cos (h \log u x)+h \sin (h \log u x)] d u \\
& -\frac{h}{4+h^{2}} \int_{1}^{\infty} \frac{S_{0}(u)+u}{u^{3}}[-h \cos (h \log u x)+2 \sin (h \log u x)] d u \\
= & \int_{1}^{\infty} \frac{S_{0}(u)+u}{u^{3}} \cos (h \log u x) d u \\
= & \int_{1}^{\infty} \frac{u-\frac{1}{2} \log u+\varepsilon(u)}{u^{3}} \cos (h \log u x) d u \\
= & \int_{1}^{\infty} \frac{1}{u^{2}} \cos (h \log u x) d u-\frac{1}{2} \int_{1}^{\infty} \frac{\log u}{u^{3}} \cos (h \log u x) d u \\
& +\frac{B}{2} \int_{1}^{\infty} \frac{1}{u^{3}} \cos (h \log u x) d u+\int_{1}^{\infty} \frac{\varepsilon(u)-B / 2}{u^{3}} \cos (h \log u x) d u \\
= & I_{1}-\frac{1}{2} I_{2}+\frac{B}{2} I_{3}+I_{4} .
\end{aligned}
$$

By an appropriate substitution and Lemma 2.7,

$$
\begin{aligned}
& I_{1}=\frac{1}{1+h^{2}} \cos (h \log x)-\frac{h}{1+h^{2}} \sin (h \log x), \\
& I_{2}=\frac{4-h^{2}}{\left(4+h^{2}\right)^{2}} \cos (h \log x)-\frac{2 h}{\left(4+h^{2}\right)^{2}} \sin (h \log x), \\
& I_{3}=\frac{2}{4+h^{2}} \cos (h \log x)-\frac{h}{4+h^{2}} \sin (h \log x) .
\end{aligned}
$$

Finally, by integration by parts,

$$
\begin{aligned}
I_{4} & =\int_{1}^{\infty} \frac{\cos (h \log u x)}{u^{3}} d f(u) \\
& =\left(1+\frac{B}{2}\right) \cos (h \log x)+\int_{1}^{\infty} \frac{f(u)}{u^{4}}[3 \cos (h \log u x)+h \sin (h \log u x)] d u
\end{aligned}
$$


because $f(1)=-1-B / 2$. Combining these results for $I_{1}, I_{2}, I_{3}, I_{4}$, we get the result.

LEMMA 6.6. We have

$$
\begin{aligned}
& \int_{0}^{1} f(u)[\cos (h \log u x)-h \sin (h \log u x)] d u \\
&=- \frac{1}{2}\left[\frac{4+3 h^{2}}{\left(4+h^{2}\right)^{2}} \cos (h \log x)+\frac{h^{3}}{\left(4+h^{2}\right)^{2}} \sin (h \log x)\right] \\
&-\left(\frac{1}{2}+\frac{B}{2}\right)\left[\frac{2+h^{2}}{4+h^{2}} \cos (h \log x)-\frac{h}{4+h^{2}} \sin (h \log x)\right] \\
&-\frac{1}{2}\left[\frac{3+h^{2}}{9+h^{2}} \cos (h \log x)-\frac{2 h}{9+h^{2}} \sin (h \log x)\right]
\end{aligned}
$$

Proof. The key is $\varepsilon(u)=\frac{1}{2} \log u-u$ when $0 \leq u \leq 1$ (see (3)). So,

$$
f(u)=\int_{0}^{u}\left[\varepsilon(v)-\frac{B}{2}\right] d v=\frac{1}{2} u \log u-\left(\frac{1}{2}+\frac{B}{2}\right) u-\frac{1}{2} u^{2} .
$$

Putting this into the integral and evaluating the integral piece by piece with suitable substitution and Lemma 2.7, one gets the result.

Proof of Theorem 1.4. First observe that when $T \leq x \leq T^{2-29 \varepsilon}$, the error term in Theorem 1.2 is $O\left(h T / \log ^{M-2} T\right)$. Rewrite Theorem 1.2 as

$$
F_{h}(x, T)=T_{1}+T_{2}+T_{3}+T_{4}+T_{5}+O\left(\frac{\tilde{h} T}{\log ^{M-2} T}\right) .
$$

Since $\frac{\sin u}{u}=1+O\left(u^{2}\right)$

$$
\begin{aligned}
& T_{3}=-\frac{T}{\pi}\left[\frac{3 \cos (h \log x)}{9+h^{2}}+\frac{h \sin (h \log x)}{9+h^{2}}\right]+O\left(T\left(\frac{T}{x}\right)^{2}\right), \\
& T_{4}=-\frac{T}{\pi}\left[\frac{\cos (h \log x)}{1+h^{2}}-\frac{h \sin (h \log x)}{1+h^{2}}\right]+O\left(T\left(\frac{T}{x}\right)^{2}\right) .
\end{aligned}
$$

By Lemma 6.5,

$$
\begin{aligned}
T_{5}= & \frac{T}{\pi} \sum_{k=1}^{\infty} \frac{\mathfrak{S}(k)}{k^{2}} \int_{0}^{1} y \cos \left(h \log \frac{k x}{y}\right) d y+O\left(T\left(\frac{T}{x}\right)^{2}\right) \\
= & \frac{T}{\pi}\left[\frac{\cos (h \log x)}{1+h^{2}}-\frac{h \sin (h \log x)}{1+h^{2}}\right] \\
& -\frac{T}{\pi}\left[\frac{4-h^{2}}{2\left(4+h^{2}\right)^{2}} \cos (h \log x)-\frac{2 h}{\left(4+h^{2}\right)^{2}} \sin (h \log x)\right]
\end{aligned}
$$




$$
\begin{aligned}
& +\frac{T}{\pi} \frac{B}{2}\left[\frac{2 \cos (h \log x)}{4+h^{2}}-\frac{h \sin (h \log x)}{4+h^{2}}\right]+\frac{T}{\pi}\left(1+\frac{B}{2}\right) \cos (h \log x) \\
& +\frac{T}{\pi} \int_{1}^{\infty} \frac{f(u)}{u^{4}}[3 \cos (h \log u x)+h \sin (h \log u x)] d u+O\left(T\left(\frac{T}{x}\right)^{2}\right) .
\end{aligned}
$$

By Lemma 4.3, (6) and Lemmas 6.2-6.4 and 6.6,

$$
\begin{aligned}
& T_{2}=-\frac{2 x \cos (h \log x)}{\pi\left(4+h^{2}\right)} \int_{1}^{\infty} \frac{\sin \frac{T y}{x}}{y^{2}} d y-\frac{4 T}{\pi} \cos (h \log x) \int_{1}^{\infty} \frac{f(y)}{y^{2}} d y \\
& +\frac{T}{\pi} \int_{1}^{\infty}\left(G_{1}(y)+G_{2}(y)\right) d y+O\left(T\left(\frac{T}{x}\right)^{1 / 2-\varepsilon}\right)+O\left(\frac{\tilde{h} T}{\log ^{M} T}\right) \\
& =-\frac{2 x \cos (h \log x)}{\pi\left(4+h^{2}\right)} \int_{1}^{\infty} \frac{\sin \frac{T y}{x}}{y^{2}} d y+O\left(\frac{\tilde{h} T}{\log ^{M} T}\right) \\
& +\frac{T}{\pi} \int_{0}^{1} f(u)[\cos (h \log u x)-h \sin (h \log u x)] d u \\
& -\frac{T}{\pi} \int_{1}^{\infty} \frac{f(u)}{u^{4}}[3 \cos (h \log u x)+h \sin (h \log u x)] d u+O\left(T\left(\frac{T}{x}\right)^{1 / 2-\varepsilon}\right) \\
& =-\frac{2 x \cos (h \log x)}{\pi\left(4+h^{2}\right)} \int_{1}^{\infty} \frac{\sin \frac{T y}{x}}{y^{2}} d y+O\left(\frac{\tilde{h} T}{\log ^{M} T}\right) \\
& -\frac{T}{\pi} \frac{1}{2}\left[\frac{4+3 h^{2}}{\left(4+h^{2}\right)^{2}} \cos (h \log x)+\frac{h^{3}}{\left(4+h^{2}\right)^{2}} \sin (h \log x)\right] \\
& -\frac{T}{\pi}\left(\frac{1}{2}+\frac{B}{2}\right)\left[\frac{2+h^{2}}{4+h^{2}} \cos (h \log x)-\frac{h}{4+h^{2}} \sin (h \log x)\right] \\
& -\frac{T}{\pi} \frac{1}{2}\left[\frac{3+h^{2}}{9+h^{2}} \cos (h \log x)-\frac{2 h}{9+h^{2}} \sin (h \log x)\right] \\
& -\frac{T}{\pi} \int_{1}^{\infty} \frac{f(u)}{u^{4}}[3 \cos (h \log u x)+h \sin (h \log u x)] d u+O\left(T\left(\frac{T}{x}\right)^{1 / 2-\varepsilon}\right) .
\end{aligned}
$$

Therefore, with miraculous cancellations,

$$
\begin{aligned}
T_{2}+T_{3}+T_{4}+T_{5}= & -\frac{2 x \cos (h \log x)}{\pi\left(4+h^{2}\right)} \int_{1}^{\infty} \frac{\sin \frac{T y}{x}}{y^{2}} d y+\frac{T}{\pi} \frac{2 B \cos (h \log x)}{4+h^{2}} \\
& +\frac{T}{\pi} \frac{4 h \sin (h \log x)}{\left(4+h^{2}\right)^{2}}+O\left(T\left(\frac{T}{x}\right)^{1 / 2-\varepsilon}\right)+O\left(\frac{\tilde{h} T}{\log ^{M} T}\right) .
\end{aligned}
$$


By Lemma 6.1 and $B=-C_{0}-\log 2 \pi$,

$$
\begin{aligned}
F_{h}(x, T)= & \frac{T}{\pi}\left[\frac{2 \cos (h \log x)}{4+h^{2}} \log x\right]-\frac{2 T \cos (h \log x)}{\pi\left(4+h^{2}\right)}\left[\frac{\sin (T / x)}{T / x}-\operatorname{ci}\left(\frac{T}{x}\right)\right] \\
& +\frac{T}{\pi} \frac{2 B \cos (h \log x)}{4+h^{2}}+O\left(T\left(\frac{T}{x}\right)^{1 / 2-\varepsilon}\right)+O\left(\frac{\widetilde{h} T}{\log ^{M-2} T}\right) \\
= & \frac{T}{\pi}\left[\frac{2 \cos (h \log x)}{4+h^{2}} \log x\right]-\frac{2 T \cos (h \log x)}{\pi\left(4+h^{2}\right)}\left[1-C_{0}-\log \frac{T}{x}\right. \\
& \left.+C_{0}+\log 2 \pi\right]+O\left(T\left(\frac{T}{x}\right)^{1 / 2-\varepsilon}\right)+O\left(\frac{\widetilde{h} T}{\log ^{M-2} T}\right) \\
= & \frac{T}{2 \pi} \log \frac{T}{2 \pi e}\left[\frac{4 \cos (h \log x)}{4+h^{2}}\right]+O\left(T\left(\frac{T}{x}\right)^{1 / 2-\varepsilon}\right)+O\left(\frac{\widetilde{h} T}{\log ^{M-2} T}\right) .
\end{aligned}
$$

7. Sketch for Conjecture 1.2. Fix $\alpha>0$. Let $r(u)$ be an even function which is almost the characteristic function of the interval $[-\alpha, \alpha]$ with $\widehat{r}(\alpha) \ll 1 / \alpha^{2}$ (see page 87 of [1] for a detailed construction). We use Conjecture 1.1 to compute the right hand side of (1):

$$
\begin{aligned}
I= & \int_{-\infty}^{\infty} F_{h}(\alpha) \widehat{r}(\alpha) d \alpha=2 \int_{0}^{\infty} F_{h}(\alpha) \widehat{r}(\alpha) d \alpha \\
= & 2(1+o(1)) \log T \int_{0}^{1} T^{-2 \alpha} \widehat{r}(\alpha) d \alpha+2 \frac{4}{4+h^{2}} \int_{0}^{1} \alpha \cos (h \log T \alpha) \widehat{r}(\alpha) d \alpha \\
& +2 \frac{4}{4+h^{2}} \int_{1}^{\infty} \cos (h \log T \alpha) \widehat{r}(\alpha) d \alpha+O\left(\frac{1}{A}\right)+o(1) \\
= & \frac{4}{4+h^{2}} \int_{-\infty}^{\infty} \cos (h \log T \alpha) \widehat{r}(\alpha) d \alpha \\
& -\frac{4}{4+h^{2}} \int_{-1}^{1}(1-|\alpha|) \cos (h \log T \alpha) \widehat{r}(\alpha) d \alpha \\
& +(1+o(1)) \log T \int_{-\infty}^{\infty} T^{-2|\alpha| \widehat{r}}(\alpha) d \alpha+O\left(\frac{1}{A}\right)+o(1) \\
= & \frac{4}{4+h^{2}} \int_{-\infty}^{\infty} \widehat{r}_{1}(\alpha) d \alpha-\frac{4}{4+h^{2}} \int_{-1}^{1}(1-|\alpha|) \widehat{r}_{1}(\alpha) d \alpha \\
& +(1+o(1)) \log T \int_{-\infty}^{\infty} T^{-2|\alpha|} \widehat{r}(\alpha) d \alpha+O\left(\frac{1}{A}\right)+o(1) \\
= & \frac{4}{4+h^{2}} I_{1}-\frac{4}{4+h^{2}} I_{2}+(1+o(1)) I_{3}+O\left(\frac{1}{A}\right)+o(1)
\end{aligned}
$$


where $r_{1}(u)=r(u+(h \log T) /(2 \pi))$. As $\int_{-\infty}^{\infty} \widehat{r}_{1}(\alpha) d \alpha=r_{1}(0)$,

$$
I_{1}=r_{1}(0)=r\left(\frac{h \log T}{2 \pi}\right) \text {. }
$$

By $\int f \widehat{g}=\int \widehat{f} g$, the transform pair and the definition of $r(u)$,

$$
\begin{gathered}
f(t)=\max (1-|t|, 0), \quad \widehat{f}(u)=\left(\frac{\sin \pi u}{\pi u}\right)^{2} \\
I_{2}=\int_{-\infty}^{\infty} r_{1}(u)\left(\frac{\sin \pi u}{\pi u}\right)^{2} d u=\int_{-\alpha+(h \log T) /(2 \pi)}^{\alpha+(h \log T) /(2 \pi)}\left(\frac{\sin \pi u}{\pi u}\right)^{2} d u+o(1) .
\end{gathered}
$$

Similarly, by the transform pair,

$$
\begin{gathered}
f(t)=e^{-2 a|t|}, \quad \widehat{f}(u)=\frac{4 a}{4 a^{2}+(2 \pi u)^{2}}, \\
I_{3}=\int_{-\alpha}^{\alpha} \frac{4 \log ^{2} T}{4 \log ^{2} T+(2 \pi u)^{2}} d u+o(1)=\int_{-\alpha+(h \log T) /(2 \pi)}^{\alpha+(h \log T) /(2 \pi)} 1 d u+o(1) .
\end{gathered}
$$

Therefore,

$$
\begin{aligned}
I= & \frac{4}{4+h^{2}} r\left(\frac{h \log T}{2 \pi}\right) \\
& +\int_{-\alpha+(h \log T) /(2 \pi)}^{\alpha+(h \log T) /(2 \pi)}\left[1-\frac{4}{4+h^{2}}\left(\frac{\sin \pi u}{\pi u}\right)^{2}\right] d u+O\left(\frac{1}{A}\right)+o(1) .
\end{aligned}
$$

Now, the left hand side of (1) is

$$
\frac{4}{4+h^{2}} r\left(\frac{h \log T}{2 \pi}\right)+\left(\frac{T}{2 \pi} \log T\right)^{-1} \sum_{\substack{0<\gamma \neq \gamma^{\prime} \leq T \\\left|\gamma-\gamma^{\prime}-h\right| \leq 2 \pi \alpha / \log T}}(1+o(1)) .
$$

Combining (16) and (17), we get Conjecture 1.2 by making $A$ arbitrarily large. The only shaky point in the above argument is the error analysis. All of these become rigorous following pages 87-90 of [1].

\section{References}

[1] T. H. Chan, Pair correlation and distribution of prime numbers, thesis, Univ. of Michigan, Ann Arbor, MI, 2002.

[2] -, On a conjecture of Liu and Ye, Arch. Math. (Basel) 80 (2003), 600-610.

[3] - More precise Pair Correlation Conjecture on the zeros of the Riemann zeta function, Acta Arith. 114 (2004), 199-214.

[4] J. B. Friedlander and D. A. Goldston, Some singular series averages and the distribution of Goldbach numbers in short intervals, Illinois J. Math. 39 (1995), 158-180. 
[5] D. A. Goldston, Large differences between consecutive prime numbers, thesis, Univ. of California, Berkeley, CA, 1981.

[6] D. A. Goldston and S. M. Gonek, Mean value theorems for long Dirichlet polynomials and tails of Dirichlet series, Acta Arith. 84 (1998), 155-192.

[7] D. A. Goldston, S. M. Gonek, A. E. Özlük and C. Snyder, On the pair correlation of zeros of the Riemann zeta-function, Proc. London Math. Soc. (3) 80 (2000), 31-49.

[8] I. S. Gradshteyn and I. M. Ryzhik, Table of Integrals, Series, and Products, 6th ed., Academic Press, San Diego, CA, 2000.

[9] H. L. Montgomery, The pair correlation of zeros of the zeta function, in: Analytic Number Theory (St. Louis, 1972), Proc. Sympos. Pure Math. 24, Amer. Math. Soc., Providence, RI, 1973, 181-193.

[10] H. L. Montgomery and R. C. Vaughan, Hilbert's inequality, J. London Math. Soc. (2) 8 (1974), 73-82.

Mathematics Department, Yost Hall 220

Case Western Reserve University

10900 Euclid Avenue

Cleveland, OH 44106-7058, U.S.A.

E-mail: tsz.chan@case.edu

Received on 22.7.2003

and in revised form on 22.6.2004 